\title{
COMUNICAÇÃO
}

\section{FLUTUAÇÃO POPULACIONAL DA MESOFAUNA EM FRAGMENTOS DE MATA NA REGIÃO DE DOURADOS-MS}

\author{
Populational fluctuation of mesofauna in fragment of native forests in Dourados - MS
}

\author{
Rosilda Mara Mussury¹, Silvana de Paula Quintão Scalon², Ana Amélia Gomes³, \\ Marcela Renata Batista ${ }^{4}$, Homero Scalon Filho ${ }^{5}$
}

\begin{abstract}
RESUMO
Como a abundância e diversidade da mesofauna edáfica em ecossistemas florestais é grande, objetivou-se, no presente estudo avaliar a flutuação e o número populacional de indivíduos da mesofauna, entre eles Acari e Collembola, em três fragmentos de matas situados na aldeia Bororó e Jaguapiru e o outro na fazenda Azulão na Região de Dourados-MS. O experimento foi conduzido em delineamento inteiramente casualizado, em esquema fatorial 3 (fragmentos) x 5 (indivíduos) x 15 (épocas de amostragem), com 10 repetições. As amostragens foram realizadas quinzenalmente, no período de fevereiro a setembro de 2005. Os indivíduos foram coletados através do funil de Berlesse modificado. Os ácaros Oribatida (Arachinida: Acari) foram os que apresentaram maiores números populacionais, diferindo estatisticamente dos Gamasida (Arachinida: Acari) e dos indivíduos Enthomobriidae (Collembola), no período de março, maio e agosto de 2005 , em todos os fragmentos analisados. O porcentual de indivíduos da fauna edáfica, nos três fragmentos, diferiram estatisticamente, sendo maior no fragmento Azulão.
\end{abstract}

Termos para indexação: Ácaros, mesofauna edáfica, flutuação populacional.

\begin{abstract}
As the abundance and diversity of soil mesofauna in forest ecosystems is large, the aim of this present study was to evaluate the fluctuation and the populational number of individuals from mesofauna, amongst them Acari and Collembola, in three parts of forests placed in Bororó and Jaguapiú tribes and another one in Azulão farm, in a region of Dourados-MS. The experiment was led in a totally random outline in factorial 3 (fragment) x 5 (individuals) x 15 (period of sampling) with 10 repetitions. The samplings were carried out fortnightly, from February to September of 2005. The individuals were collected through a modified Berlesse funnel. Individuals Oribatida (Arachinida: Acari) were those which presented the highest populational numbers, differing statistically from Gamasida (Arachinida: Acari) and individuals Enthomobriidae (Collembola) during march, may and august of 2005, in all analysed parts. The individuals percentage from soil fauna in the three parts differed themselves statistically, being higher in Azulão area.
\end{abstract}

Index terms: Acari, edaphic mesofauna, populational fluctuation.

\section{(Recebido em 31 de julho de 2006 e aprovado em 14 de fevereiro de 2007)}

De acordo com Wallwork (1970), a abundância e diversidade da mesofauna edáfica em ecossistemas florestais é grande e, conforme Beare et al. (1995), Butcher \& Snider (1971), Lavelle et al. (1995) e Seasted (1984), importantes para os processos de decomposição e mineralização da matéria orgânica, influenciando dessa forma, os níveis locais de fertilidade e todo o funcionamento dos ecossistemas terrestres (WITKAMP, 1971).

Segundo Rusek \& Marshall (2000) os microartrópodes podem ser indicadores valiosos dos impactos antropogênicos em florestas, bem como em diversos agroecossistemas conforme ressaltado por Behan-Pelletier (1999) e Mussury et al. (2002).

No Mato Grosso do Sul, constatou-se que técnicas agrícolas convencionais (MUSSURY et al., 2002) e a fragmentação de matas em áreas indígenas (GOMES et al., 2007) geram entre outros fatores, reduções populacionais da mesofauna edáfica. Nessa visão, Laurence et al. (2002) afirmam que a crescente fragmentação dos ecossistemas florestais é um dos grandes problemas ambientais do mundo

${ }^{1}$ Bióloga, Doutora - Faculdade de Ciências Biológicas e Ambientais/FCBA - Universidade Federal da Grande Dourados/UFGD - Rodovia Dourados, Itahum, Km 12 - Cx. P. 533 - 79804-970 - Dourados, MS - mara.mussury@ufgd.edu.br

${ }^{2}$ Bióloga, Doutora - Faculdade de Ciências Agrárias/FCA - Universidade Federal da Grande Dourados/UFGD - Rodovia Dourados, Itahum, Km 12 - Cx. P. 533 - 79804-970 - Dourados, MS - silvana.scalon@ufdg.edu.br

${ }^{3}$ Especialista - Centro Universitário da Grande Dourados/UNIGRAN - Rua Balbina de Matos, 2121 - 79824-900 - Dourados, MS - anagomes@unigran.br ${ }^{4}$ Bióloga - Centro Universitário da Grande Dourados/UNIGRAN - Rua Balbina de Matos, 2121 - 79824-900 - Dourados, MS - marcelarenata11@yahoo.com.br ${ }^{5}$ Mestre - Universidade Estadual de Mato Grosso do Sul/UEMS - Rodovia Aquidauana/CERA, Km 12 - Cidade Universitária - $79800-000$ - Aquidauana, MS - homero@uems.br 
moderno, sendo freqüentemente relacionada a efeitos deletérios sobre as comunidades bióticas. Tais efeitos deletérios estão intimamente relacionados à flutuação populacional dos indivíduos.

Para Wallwork (1971), a flutuação populacional dos indivíduos da mesofauna, relaciona-se com a duração do ciclo biológico e o número de gerações anuais das espécies constituintes. Esse mesmo autor comenta que, nos Estados Unidos e na Europa, os Cryptostigmata (Arachinida: Acari) apresentam picos de populações nos meses de inverno e de outono e menores no verão, sugerindo assim, que isso seja atribuído ao fato de os adultos depositarem seus ovos no final do verão, conseqüientemente as formas jovens aparecerem em grande quantidade no inverno.

Conforme Brady (1989) muitos grupos de artrópodes do solo têm mais importância porque são amplamente distribuídos, ou porque suas atividades tenham maior efeito sobre o solo, vegetação ou sobre os demais membros da fauna.

Objetivou-se, nesse estudo avaliar a flutuação e o número populacional de indivíduos da mesofauna, entre eles Acari e Collembola, em três fragmentos de matas na Região de Dourados, MS, no período de fevereiro a setembro de 2005.

Esta pesquisa foi conduzida no período de fevereiro a setembro de 2005 em três fragmentos florestais, sendo dois deles situados a, aproximadamente, cerca de cinco km do município de Dourados, MS (22¹3'16" de latitude Sul e $54^{\circ} 48^{\prime} 2^{\prime \prime}$ de longitude Oeste e tem altitude de $452 \mathrm{~m}$ ), formados pelas aldeias Jaguapiru e Bororó (22¹0'22' $\mathrm{S}$ e $\left.54^{\circ} 49^{\prime} 05^{\prime} \mathrm{W}\right)$ e o outro localizado na fazenda Azulão (22¹1'57'S e 5454'29' W), a cerca de 10 km do município.

No período de coleta, a mesofauna foi amostrada quinzenalmente em cada área. Utilizou-se o funil de Berlesse modificado numa faixa de zero a cinco centímetros de profundidade e com um volume de solo de $250 \mathrm{~cm}^{3}$. As amostras de solo foram coletadas em pontos específicos na área central da mata e em zigue-zague. As amostras foram acondicionadas em sacos plásticos, para minimizar as perdas de umidade, e em seguida instaladas em mesas expositoras, cuja fonte de luz e calor foram lâmpadas de $25 \mathrm{w}$, permanecendo por sete dias no laboratório do Centro Universitário da Grande Dourados - UNIGRAN.

Os indivíduos foram recolhidos em potes plásticos, contendo a seguinte solução: $75 \%$ de álcool, $23 \%$ de água destilada e $2 \%$ de glicerina. Após o período de exposição foram identificados, contados e separados os indivíduos, com auxílio de um microscópio estereoscópico Lambda.

$\mathrm{O}$ experimento foi conduzido em delineamento inteiramente casualizado, em esquema fatorial 3 (fragmentos) x 5 (indivíduos) x 15 (épocas de amostragem), com 10 repetições. Os dados originais foram transformados para $\sqrt{\mathrm{X}+0,5}$ e as médias comparadas pelo teste Tukey (SNEDECOR \& COCHRAN, 1989).

Os dados climatológicos foram fornecidos pela Empresa de Pesquisa Agropecuária Oeste-EMBRAPA, em Dourados, localizadas na rodovia BR 163, Km 253, trecho Dourados-Caarapó-MS e constam na Figura 1.

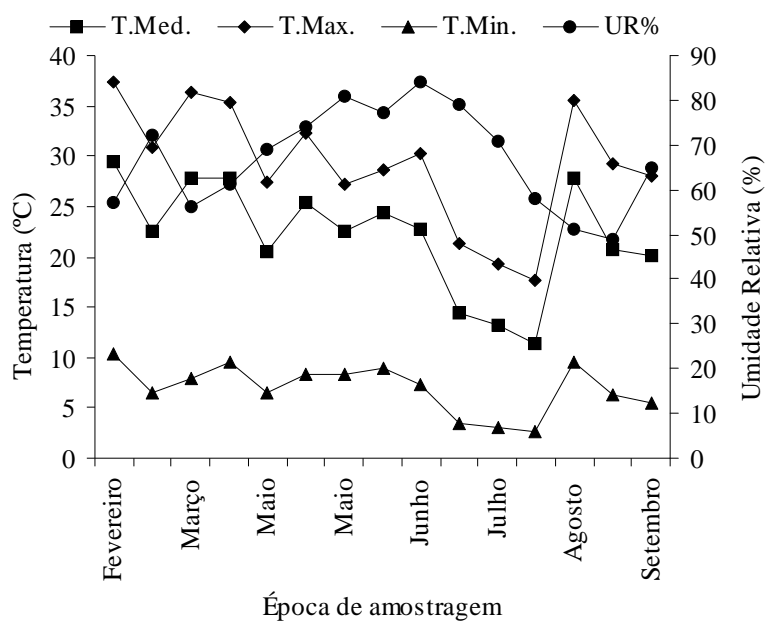

Figura 1 - Dados climatológicos obtidos no período de fevereiro a setembro de 2005. (T. Méd. - temperatura média, T. Max. - temperatura máxima, T. Min. - temperatura mínima, UR - umidade relativa. Dourados-MS. 2005 (Fonte: Embrapa CPAO).

A análise de variância obtida a partir dos dados coletados está representada na Tabela 1 .

O número médio de ácaros Oribatida (Arachinida: Acari) e Gamasida (Arachinida: Acari) e Enthomobriidae (Collembola) coletados nos fragmentos de matas observamse na Figura 2. Observa-se que, em média (Figura 2), a maior densidade populacional de indivíduos da mesofauna está representada pelos Oribatida (Arachinida: Acari), diferindo estatisticamente dos Gamasida (Arachinida: Acari) e dos Entomobriidae (Collembola).

$\mathrm{O}$ fragmento Azulão apresentou o maior número médio de indivíduos, seguido do fragmento Jaguapirú e do Bororó, em diferentes meses do ano (Figuras 3).

De acordo com as observações "in loco", e segundo Pott \& Pott (2003) a vegetação dos três fragmentos é classificada como mata mesófila semidecídua, caracterizandose pela mistura de espécies caducifólias e perenifólias. Os fragmentos aparentemente apresentam-se em vários estádios de degradação e regeneração, especialmente os fragmentos localizados na Reserva Indígena, que se apresentam mais 
Tabela 1 - Dados da análise de variância da população de artrópodes de solo considerando a época e o local de coleta. Dourados-MS. 2005.

\begin{tabular}{lcc}
\hline \multicolumn{1}{c}{ Causas da Variação } & GL & Quadrado médio \\
\hline Fragmento & 2 & $44,4697^{* *}$ \\
Indivíduos & 4 & $1707,1538^{* *}$ \\
Época & 14 & $160,9909^{* *}$ \\
Fragmento x Indivíduos & 8 & 14,2031 \\
Fragmento x Época & 28 & 13,8807 \\
Indivíduos x Época & 56 & 72,1602 \\
Fragmento x Indivíduos x Época & 112 & 8,2045 \\
Resíduo & 2025 & 4,2236 \\
\hline
\end{tabular}

** Significativo ao nível de $1 \%$ de probabilidade.

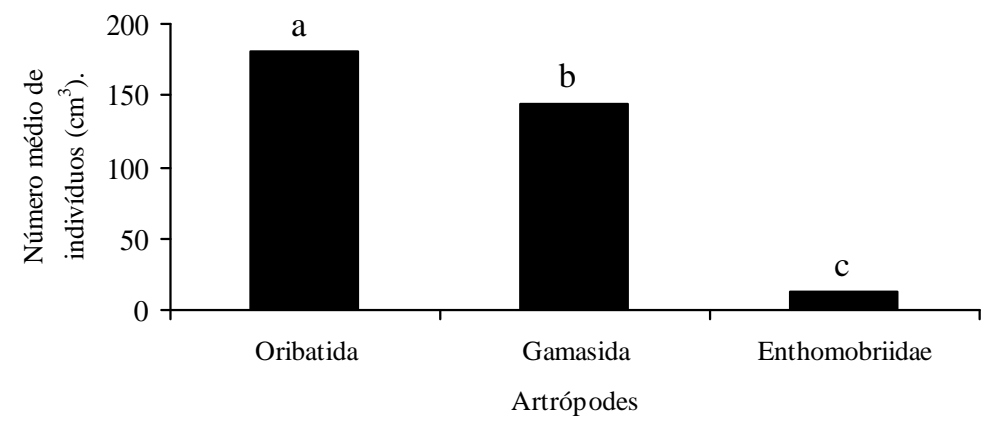

Figura 2 - Número médio de indivíduos, capturados nos três fragmentos de mata (Azulão, Bororó e Jaguapirú). As médias foram comparadas pelo teste Tukey, a 5\% de probabilidade. Dourados-MS. 2005.

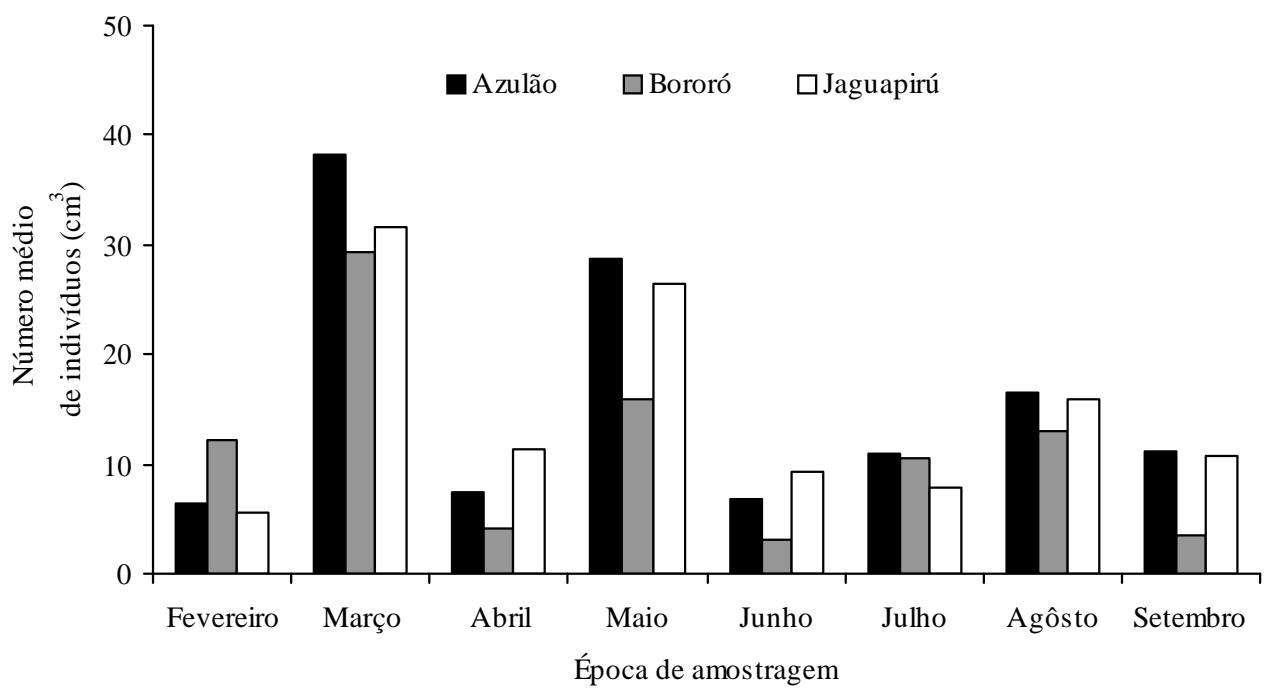

Figura 3 - Número médio de indivíduos coletados em diferentes meses do ano nos fragmentos Azulão, Bororó e Jaguapirú. Dourados-MS. 2005. 
degradados pela ação antrópica, como extração de madeira, principalmente de espécies vegetais do subdossel para obtenção de lenha e pelo impacto do cultivo de monoculturas, além do desmatamento que cada vez mais fragmenta os habitats remanescentes. O fragmento Azulão, mesmo sendo uma área que também sofre com o impacto das lavouras de monocultura como soja, aveia e milho que a cerca, parece ser a área melhor preservada por causa do sua maior extensão contínua de área de mata.

No fragmento Bororó as perturbações foram mais intensas, o fluxo de índios e animais decorreu em menor densidade populacional da mesofauna edáfica, corroborando com os resultados observados por Gomes et al. (2007), nessa mesma área. O porcentual de indivíduos da fauna edáfica, nos três fragmentos diferiram estatisticamente, sendo maior no fragmento Azulão, seguido do Jaguapirú e Bororó (Figura 4).

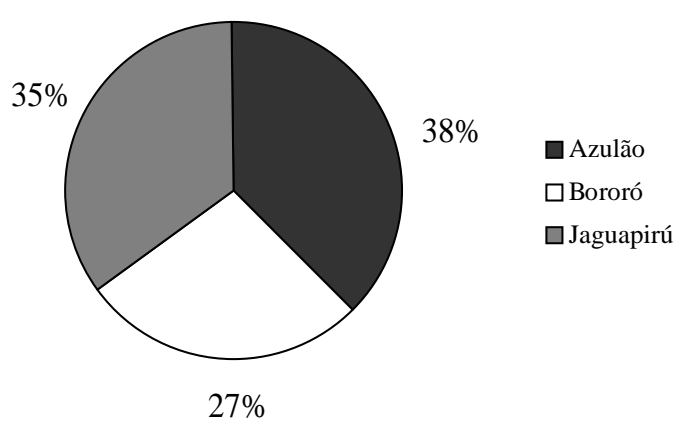

Figura 4 - Porcentual de indivíduos da fauna edáfica encontrados nas três áreas estudadas no período de fevereiro a setembro de 2005. Dourados-MS.

Nos três fragmentos os Oribatida (Arachinida: Acari) foram predominantes, diferindo estatisticamente dos Gamasida (Arachinida: Acari) e dos Enthomobriidae (Collembola) (Figura 2 e 5 A, B, C). No entanto, vale ressaltar que, o maior porcentual de indivíduos Oribatida observados foi no fragmento Bororó (Figura 5B).

Conforme ressaltado por Sautter et al. (1994) e Tousignant \& Coderre (1992) de forma geral a mesofauna edáfica é formada principalmente por ácaros Oribatida que demonstram uma maior preferência por habitats ricos em matéria orgânica, além de apresentarem habilidades em serem os primeiros colonizadores de áreas perturbadas (SAUTER \& SANTOS, 1994).

Corroborando também com os dados apresentados, Bitencourt et al. (2006) e Mussury et al. (2002) observaram que, em ambientes amplamente manejados, quer por pisoteio de gado ou uso de maquinário pesado ocorre uma menor diversidade e abundância de organismos da mesofauna.
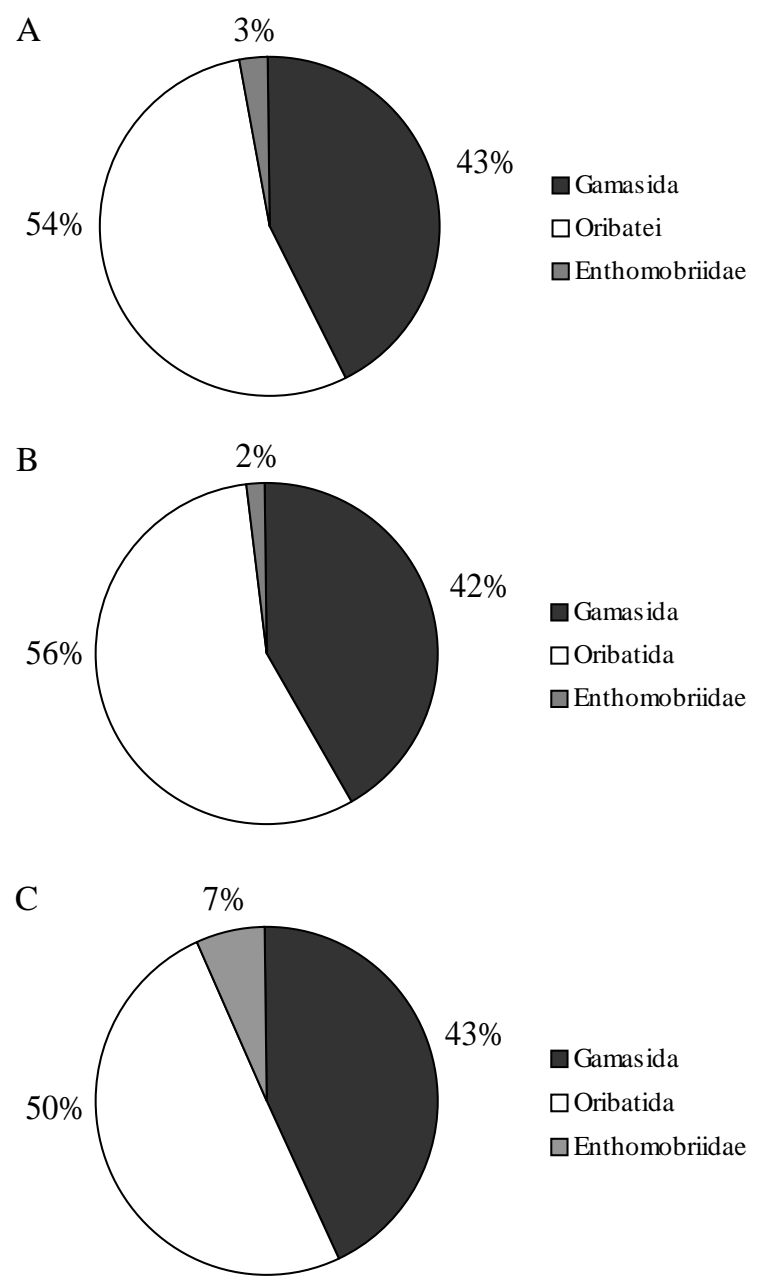

Figura 5 - Porcentual de indivíduos da fauna edáfica encontrados nas três áreas estudadas no período de fevereiro a setembro e 2005. A-Azulão, B-Bororó e CJaguapirú. Dourados-MS. 2005.

A flutuação de Oribatida, Gamasida e Enthomobriidae está representada na Figura 6. Poucos representantes Poduridae e Sminthuridae foram encontrados, por isso não estão representados nas Figuras.

De acordo com Hodkinson et al. (1994) os Collembola são muito sensíveis à dessecação e perda de água cuticular, o que aliado à ausência de cobertura vegetal e alta temperatura do solo, resulta em uma população de Collembola reduzida. Esse fato pode ser observado na Figura 5B para o fragmento Bororó o mais perturbado pelo pisoteio de índios, animais e retirada de árvores.

Durante o mês de março, maio e agosto (Figura 3), em todos os fragmentos analisados foram observados os maiores números médios de indivíduos encontrados, sendo 
Oribatida predominantes (Figura 6). O aumento no número de indivíduos ocorridos em março, maio e agosto, nos três fragmentos estudados, pode ser decorrente, entre outros fatores, da temperatura em torno de 25 a $28^{\circ} \mathrm{C}$ e umidade relativa de $56 \%$ a $74 \%$. Observou-se redução no número médio populacional nos meses de abril e junho (Figura 3 e 6) provavelmente, tal fator possa ser decorrente das quedas na temperatura ambiental registradas no período (Figura 1).

Para explicar a flutuação populacional dos indivíduos da mesofauna é muito difícil isolar um ou outro fator abiótico, pois eles se interagem. No entanto, outros fatores podem e devem ser considerados, como o movimento humano ou de animais na área que desloca o material vegetal acumulado, expondo os indivíduos e o solo e dessa forma, reduzindo a população dos indivíduos.

Essa explicação pode justificar os resultados observados no fragmento Bororó, pois animais como Collembola não têm proteção pigmentar suficiente para suportar os raios solares diretos, diminuindo conseqüentemente a população desses indivíduos (PRIMAVESI, 1990; RICHARDS, 1974). Conforme Butcher \& Snider (1971) a redução da precipitação na região por um longo período de estiagem e uma baixa umidade podem resultar em migração, baixa reprodução e alta mortalidade.

Dessa forma, pode-se concluir que os maiores porcentuais de indivíduos foram observados nas matas dos fragmentos da fazenda Azulão e Jaguapirú; os ácaros (Arachinida: Oribatida) foram os que apresentaram maiores números populacionais, seguidos dos Gamasida e dos Enthomobriidae (Collembola) no período de março, maio e agosto de 2005, em todos os fragmentos analisados.

\section{AGRADECIMENTOS}

À UNIGRAN (Centro Universitário da Grande Dourados) a oportunidade de realização da pesquisa, à FUNDECT-MS (Fundação de apoio ao desenvolvimento do Ensino, Ciência e Tecnologia do Estado de Mato Grosso do Sul), o apoio financeiro.
A

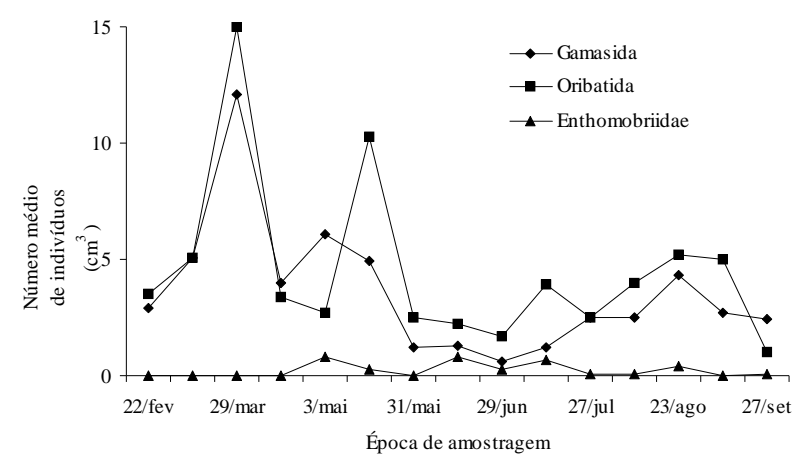

B

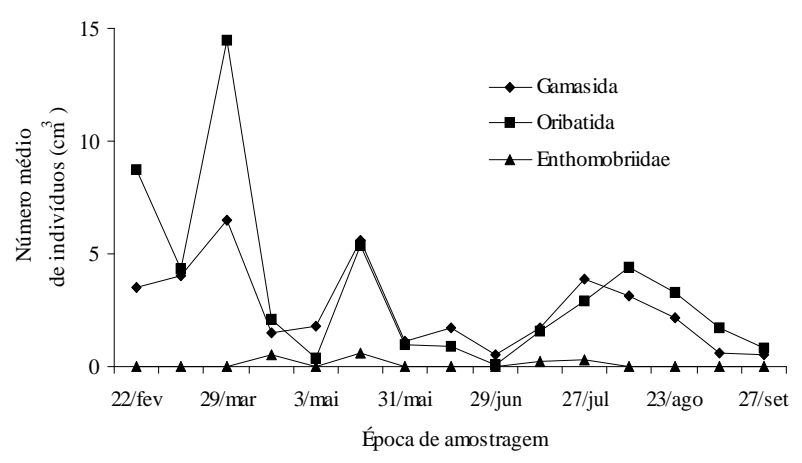

C



Figura 6 - Flutuação populacional de Acari e Collembola, amostrados durante o período de fevereiro a setembro de 2005, nos fragmentos Azulão (A), Bororó (B) e Jaguapirú (C), em Dourados-MS. 2005. 


\section{REFERÊNCIAS BIBLIOGRÁFICAS}

BEARE, M. H.; COLEMAN, D. C.; CROSSLEY, D. A.; HENDRIX, P. F.; ODUM, E. P. A hierechical approach to evaluating the significance of soil biodiversity the biogeochemical cycling. Plant and soil, The Hague, v. 170, p. 5-22, 1995.

BEHAN-PELLETIER, V. M. Oribatid mite biodiversity in agroecoystems: role for bioindication. Agriculture, Ecosystem and environment, Amsterdam, v. 74, p. 411-423, 1999.

BITENCOURT, D. R.; CARVALHO, E. da S. M.; SILVA, C. B. da S.; SANTOS, H. R. dos. Levantamento da fauna edáfica em diferentes ambientes na região do Pantanal SulMatogrossense, Corumbá-MS. In: FERTBIO, 2006, Bonito, MS. Anais... Bonito: FertBio, 2006.

BRADY, N. C. Natureza e propriedades dos solos. 7. ed. Rio de Janeiro: F. Bastos, 1989.

BUTCHER, J. W.; SNIDER, R. J. Bioecology of edaphic Collembola and Acarina. Annual Review of Entomology, Palo Alto, v. 16, p. 249-288, 1971.

GOMES, A. A.; MUSSURY, R. M.; SCALON, S. P. Q.; WATHIER, F.; CUNHA, K. A. A.; SCALON-FILHO, H. Avaliação do impacto da fragmentação de florestas nativas sobre a mesofauna edáfica na região de Dourados-MS. Ciência e Agrotecnologia, Lavras, v. 31, n. 3, p. 612-618, 2007.

HODKINSON, I. D.; HEALEY, V.; COULSON, S. Moisture relantioships of the high artc collembolan onychiurus arcticus. Physiological Entomology, London, v. 19, p. 109114, 1994.

LAVELLE, P.; LATTAUD, C.; TRIGO, D.; BAROIS, I. Mutualism and biodiversity in soils. Plant and soil, The Hague, v. 170, p. 23-33, 1995.

LAURANCE, W. F.; LOVEJOY, T. E.; VASCONCELOS, H. L.; BRUNA, E. M.; DIDHAM, R. K.; STOUFFER, P. C.; GASCON, C.; BIERREGAARD, R. O.; LAURANCE, S. G.; SAMPAIO, E. Ecosystem decay of Amazonian forest fragments: a 22-year investigation. Conservation Biology, Boston, v. 13, n. 3, p. 605-618, 2002.
MUSSURY, R. M.; SCALON, S. P. Q.; SILVA, S. V. da; SOLIGO, V. R. Study of acari and collembola population in four cultivation systems Dourados, MS. Brazilian Archives of Biology and Technology, São Paulo, v. 45, n. 3, p. 257-264, 2002.

POTT, A.; POTT, V. J. Espécies de fragmentos florestais em Mato Grosso do Sul. In: COSTA, R. B. (Org.). Fragmentação florestal e alternativas de desenvolvimento rural na região Centro-Oeste. Campo Grande: UCDB, 2003. p. 26-52.

PRIMAVESI, A. Manejo ecológico do solo: agricultura em regiões tropicais. 9. ed. São Paulo: Nobel, 1990. p. 142-154.

RICHARDS, B. N. Introduction to the soil ecosystem. New York: Longmam, 1974.

RUSEK, J.; MARSHALL, V. G. Impact of airborne pollutantes on soil fauna. Annual Review of Ecology and systematics, Palo Alto, v. 31, p. 395-423, 2000.

SAUTER, K. D.; SANTOS, H. R. dos. Avaliação da estrutura da população da mesofauna edáfica, em diferentes regimes de reabilitação de um solo degradado pela mineração do xisto. Revista de Ciências Agrárias, Curitiba, v. 13, n. 1/2, p. 31-34, 1994.

SEASTED, T. R. The role of microarthropods in decomposition and mineralization processes. Annual Review of Entomology, Palo Alto, v. 29, p. 25-46, 1984.

SNEDECOR, G. W.; COCHRAN, G. W. Statistical methods. 8. ed. Lowa: State University, 1989

TOUSIGNANT, S.; CODERRE, D. Niche partitioning by soil mites in a recent hardwood plantation in Southern Quebec, Canada. Pedobiologia, Ottawa, v. 36, p. 287-294, 1992.

WALLWORK, J. A. Ecology of soil animals. London: McGraw-Hill, 1970.

WALLWORK, J. A. Acari. In: BURGES, A.; RAW, F. Biologia del suelo. Barcelona: Omega, 1971. p. 425-459.

WITKAMP, B. Soils as components of ecosystems. Ecology Systematics, [S.1.], v. 2, p. 85-110, 1971. 